\title{
High Resolution Ultrasound in Cutaneous and Subcutaneous Lesions
}

\author{
A. Einstien ${ }^{1}$, Aniruddha P Rangari ${ }^{2}$, Meghanaa Jaya Kumar ${ }^{3}$, Dinisha Einstien ${ }^{4}$ \\ ${ }^{1}$ Associate Professor, Department of Radiology and Imaging Science, Chettinad Hospital and Research Institute, Chennai, \\ ${ }^{2}$ Consultant, Department of Radiologist, Indian Scan, Chennai, ${ }^{3}$ Consultant, Department of Radiologist, Chennai, ${ }^{4}$ Assistant \\ Professor, Department of Pathology, A.C.S. Medical College and Hospital, Chennai, India
}

Corresponding author: Dr. Aniruddha P Rangari, 1, V.G.P. Pushpa Nagar Extn., Medavakkam, Chennai - 600 100, India

DOI: http://dx.doi.org/10.21276/ijcmsr.2018.3.4.15

How to cite this article: A. Einstien, Aniruddha P Rangari, Meghanaa Jaya Kumar, Dinisha Einstien. High resolution ultrasound in cutaneous and subcutaneous lesions. International Journal of Contemporary Medicine Surgery and Radiology. 2018;3(4):D66-D70.

\section{A B S T R A C T}

Introduction: In the evaluation of cutaneous lesions, High Resolution Ultrasonography (HRUS) with color Doppler is useful as a safe, noninvasive, economical, and repeatable diagnostic procedure that can reduce and replace invasive procedures like fine needle aspirations and biopsies. Analysis of the skin surface allows the investigation of tumor and inflammatory diseases, the measurement of skin thickness. The objective of this study was to evaluate the role of HRUS in cutaneous lesions with histopathological correlation wherever possible.

Material and Methods: A prospective study of 100 patients with skin lesions based on clinical data were subjected to HRUS examination. Collected data was analysed by descriptive statistics.

Results: The study comprised of a total of 100 patients, out of which 75 were non-neoplastic and 25 were neoplastic lesions. The non-neoplastic lesions included Psoriasis, edema, lymph nodes and vascular malformations. The neoplastic lesions included 16 benign and 9 malignant cases. The observed HRUS and Doppler characteristics of these lesions are discussed in detail.

Conclusion: HRUS is non-invasive, affordable, easily available, rapid imaging technology. It can be used to localize the skin lesion, generate differential diagnosis and to follow up known lesion.

Key words: High Resolution Ultrasonography, Cutaneous Lesions, Psoriasis, Vascular Malformations, Benign, Malignant

\section{INTRODUCTION}

Ultrasound imaging has been used in dermatology for nearly 30 years. In 1979, Alexander and Miller first introduced ultrasonography (USG) as a noninvasive technique to measure normal skin thickness, and in the 1980s and 1990s, highresolution ultrasonography (HRUS). ${ }^{1}$ The requirements for the noninvasive ultrasonic investigations of human skin from the dermatologist point of view are defined as it is expected to determine the size, contour, structure, penetration, depth of skin lesions. In the evaluation of cutaneous lesions, HRUS with color Doppler is useful as a safe, noninvasive, economical, and repeatable diagnostic procedure that can reduce and replace invasive procedures like fine needle aspirations and biopsies. ${ }^{1}$ Ultrasonography with frequency more than $7 \mathrm{MHz}$ allows the observation of the skin and its appendages, subcutaneous tissue. The development of devices with frequencies above $15 \mathrm{MHz}$ allowed the differentiation of skin layers, increasing considerably its use in dermatology. By analyzing the skin surface in three dimensions, the HFUS, associated with color Doppler, allows the investigation of tumor and inflammatory diseases, the measurement of skin thickness. ${ }^{2}$ Color Doppler is able to increase significantly the specificity of sonography in the evaluation of skin nodules. ${ }^{3}$
High resolution ultrasonography allows the identification of macular and nodular pigmentary lesions located up to $1.5 \mathrm{~cm}$ in depth. Cutaneous ultrasound can be used as screening method for pigmentary lesions while providing valuable information concerning therapeutic approach. High resolution ultrasonography, using $20-50 \mathrm{MHz}$ transducers, is a novel diagnostic procedure for the investigation of the skin and its specific conditions. ${ }^{4}$ The objective of this study was to evaluate the role of HRUS in cutaneous lesions with histopathological correlation wherever possible.

\section{MATERIAL AND METHODS}

A prospective study of 100 patients in all age groups with skin lesions based on clinical data who presented to Bowring and Lady Curzon Hospital, Victoria Hospital and Vani Vilas hospital attached to Bangalore Medical College and Research Institute, Bengaluru, from November 2014 to May 2015 were subjected to HRUS examination.

Study design: It was a Cross sectional study. Totally 100 patients were studied from November 2014 to May 2016. Inclusion criteria: All clinically suspected patients with superficial skin lesions. Exclusion Criteria: Patients with trauma. After taking Informed consent, all patients included 
in the study underwent 2D grey scale real time HRUS of skin lesion, using a linear probe of 7-20 MHZ coupled with colour Doppler equipment, in Philips IU22 ultrasound machine, and characteristics of the lesion were assessed. Compression was avoided in superficial lesions because this may result in a false thinning or superficial nodules might move outside the field of view. ${ }^{5}$

\section{Specifications of the ultrasound machine}

Manufacturer: Philips. Model: IU22.

Image storage: Hard disc.

Type of transducer: Linear array. Frequency of transducer: $7-20 \mathrm{MHz}$

\section{STATISTICAL ANALYSIS}

Collected data was analysed using descriptive statistics (Percentages and proportions).

\section{RESULTS}

The study comprised of a total of 100 patients, out of which 75 were non-neoplastic and 25 were neoplastic lesions. The non-neoplastic lesions included Psoriasis (29 cases), edema (21 cases),lymph nodes (15 cases) and vascular malformations (10 cases). The neoplastic lesions included 16 benign and 9 malignant cases. Lipomas were the most common (12 cases). The other neoplastic lesions included Hemangioma (3 cases), neurofibroma (1 case), basal cell carcinoma (6 cases) and melanoma (3 cases).

In the present study, hypoechoic skin lesions were most common, followed by hyperechoic lesions and then anechoic lesions. [Table -1 , figure 1-5]
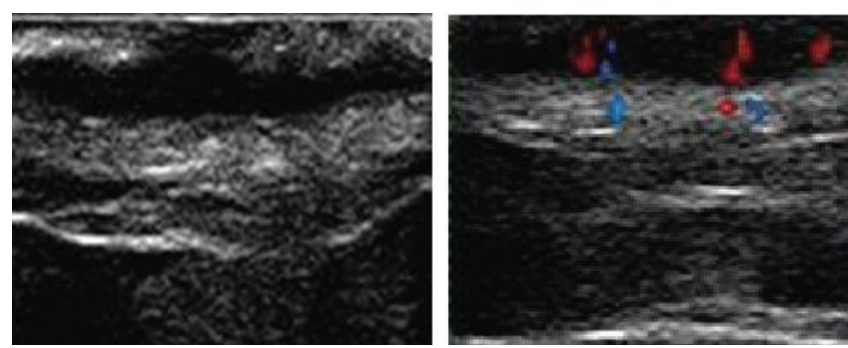

Figure-1: Fairly defined hypoechoic lesion with internal vascularity, consistent with BCC (Grey scale and color Doppler image)
Benign tumors showed minimal internal vascularity, whereas the malignant tumors showed increased vascularity. On the other hand, lymphatic malformations showed no significant vascularity. [Table -2]

HRUS of psoriatic skin showed thickened epidermis and dermis, absence of involvement of the subcutaneous tissue and increased vascularity on Doppler study. All 21 patients in the present study with edema showed decrease in echogenicity with minimal internal vascularity. In the present study $15 \%$ of the skin lesions were lymph nodes. In
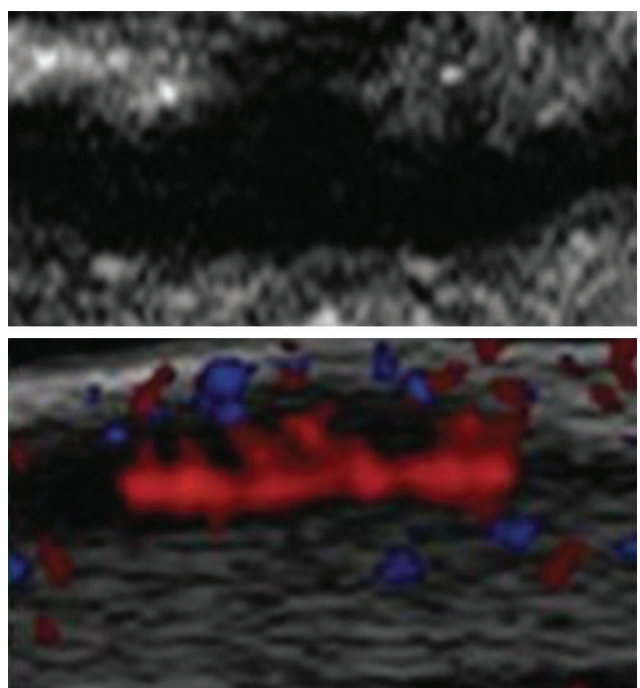

Figure-2: Fairly defined heterogenous predominantly hypoechoic lesion with internal vascularity,consistent with melanoma (Grey scale and color Doppler image)
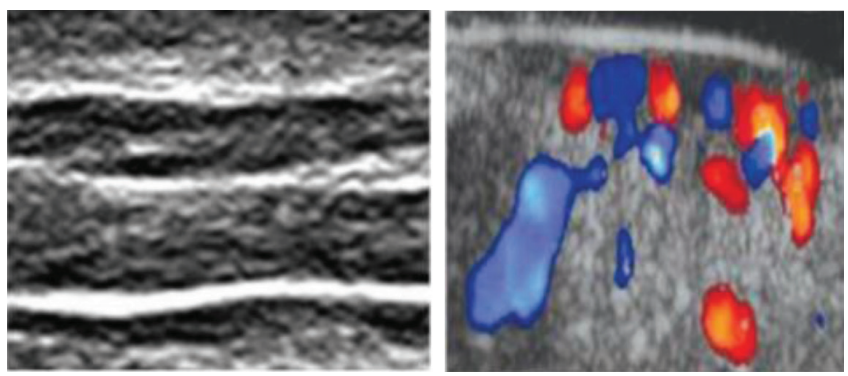

Figure-3: Thickening of the skin with mild increase in echogenicity, consistent with psoriasis (Grey scale, color Doppler)

\begin{tabular}{|l|c|c|c|c|c|c|c|c|c|}
\hline Echogenicity & Psoriasis & Edema & Lymph node & VM & Lipoma & HMG & NF & BCC & Melanoma \\
\hline Hyperechoic & 29 & - & - & - & 12 & - & - & - & - \\
\hline Hypoechoic & - & 21 & 15 & - & - & 3 & 1 & 6 & 3 \\
\hline Isoechoic & - & - & - & - & - & - & - & - & - \\
\hline Anechoic & - & - & - & 10 & - & - & - & - & - \\
\hline Heterogenous & - & - & - & - & - & - & - & - & - \\
\hline \multicolumn{7}{|l}{ Table-1: Distribution of cases according to echogenicity } \\
\hline
\end{tabular}

\begin{tabular}{|l|c|c|c|c|c|c|c|c|c|}
\hline Vascularity & Psoriasis & Edema & Lymph Node & VM & Lipoma & HMG & NF & BCC & Melanoma \\
\hline Increased & 29 & - & - & $3(\mathrm{~A})$ & - & 3 & - & 6 & 3 \\
\hline Minimal & - & 21 & 12 & $4(\mathrm{~V})$ & 12 & - & 1 & - & - \\
\hline No & - & - & 3 & $3(\mathrm{~L})$ & - & - & - & - & - \\
\hline VM - Vascular Malformation, HMG - Hemangioma, NF - Neurofibroma, BCC - Basal Cell Carcinoma \\
\hline \multicolumn{8}{|l|}{ Table-2: Distribution of cases according to the vascularity } \\
\hline
\end{tabular}



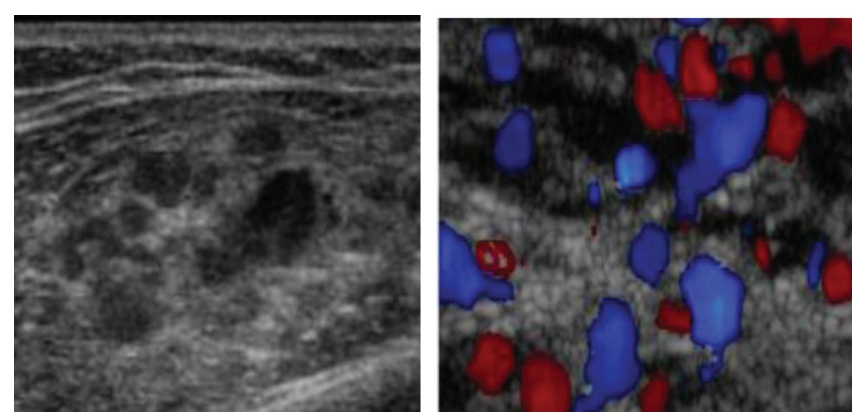

Figure-4: Fairly defined hypoechoic mass with anechoic cystic spaces within showing vascularity and monophasic flow on spectral Doppler, consistent with venous malformation. (Grey scale and color Doppler image)

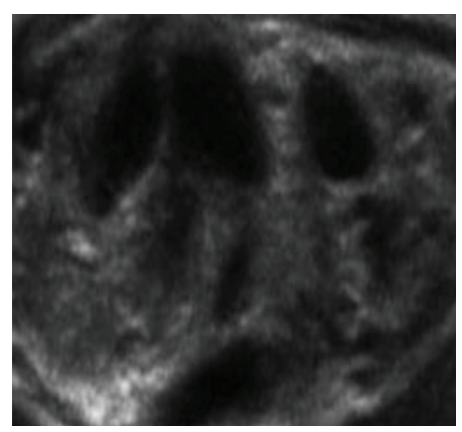

Figure-5: Fairly defined hypoechoic lesion with anechoic spaces within with no internal vascularity, consistent with lymphatic malformation. (Grey scale image)

pathological conditions the acoustic impedance of lymph nodes becomes different from that of normal skin making them visible. In the present study, out of 15 patients with lymph nodes, 13 were inflammed with loss of fatty hilum. However, just the absence of fatty hilum cannot be used to confirm the diagnosis, so further HPE correlation is needed. In the present study, most of the vascular malformations were venous (4), followed by 3 arterial and 3 lymphatic.

Lipomas appeared hyperechoic on HRUS with minimal internal vascularity. All three cases of hemangioma showed heterogenous echopattern and hypervascularity. The single case of neurofibroma studied was hypoechoic on HRUS with minimal internal vascularity. 6 cases of BCC were studied and were found to be hypoechoic on HRUS and all showed increased vascularity. HRUS findings of melanoma included hypoechoic lesion which showed increased vascularity.

HPE correlation was available for 42 out of the 100 cases. $\mathrm{HPE}$ was performed for all cases of BCC, melanoma, lipoma, lymph nodes and neurofibroma, however out of 29 patients of psoriasis studied, HPE was performed in only 5 cases.

\section{DISCUSSION}

HRUS is an invaluable tool for imaging in dermatology. It can provide crucial preoperative information by serving as a firstline examination for both thickness and characterization of cutaneous malignant melanoma. Compared with a physical examination, HRUS is very effective in the early detection of lymph node metastases. ${ }^{6}$

HRUS of the normal skin shows epidermis as a thin continuous hyperechoic layer with uniform thickness, while the dermis appears relatively less echoic and subcutaneous tissue is characteristically hypoechoic. Main HRUS feature of psoriatic skin are thickened epidermis and dermis, absence of involvement of the subcutaneous tissue and increased vascularity on Doppler study. ${ }^{7}$

On sonography, edema usually appears as anechoic fluid between the lobules of the subcutaneous tissue. In cases with lymphedema there is thickening of all the cutaneous layers, hypoechogenicity of the dermis, and increased echogenicity of the subcutaneous tissue in addition to the anechoic fluid between the fatty lobules of the hypodermis. ${ }^{8,9}$

Echogenic hilus is a normal sonographic feature of most of the normal cervical lymph nodes (86\%), and it is commonly seen in larger nodes. ${ }^{10}$ Although metastatic, lymphomatous and tuberculous nodes tend to have absent hilus, they may present with an echogenic hilus in their early stage of involvement in which the medullary sinuses have not been sufficiently disrupted to eradicate it. ${ }^{10,11,12}$ Therefore, the presence/absence of echogenic hilus should not be the sole criterion in the diagnosis.

The presentation of arterial and AVMs on B-Mode ultrasound is quite variable. Typically located superficially in the subcutaneous tissue, they may result in thickening of the skin and subcutis. A nest of anechoic ducts can usually be detected, even though in some cases an anechoic pseudocystic appearance can be encountered. Additionally, a mixed pattern composed by hyperechoic vascular stroma and anechoic vascular channels of different size and diameter may be found. The overall appearance of AVMs can be quite inhomogeneous, especially because of the different amount of arterial and venous elements inside the lesion. Typically arterial malformations and AVMs do not have a soft-tissue component. AVMs often have indistinct outer borders and sometimes the vessels inside an AVM are so small that they are hardly seen with B-Mode ultrasound, and only detected when color Doppler ultrasound is applied. 8,13

Venous malformations are anechoic or slightly hypoechoic, sponge-like structures on B-mode sonograms and they consist of multiple blood-filled caverns of different sizes. Typically venous malformations have only little fibrous stroma, but the walls of the individual caverns range from very thin septae to thick fibrous bridges, which is why approximately $80 \%$ of venous malformations are somewhat inhomogeneous on B-mode with a mixture of hypoechoic caverns and sometimes hyperechoic septations. Tiny roundish calcifications resembling phleboliths inside thrombosed caverns are a diagnostic hallmark of venous malformations but are seen in only $20 \%$ of lesions. A color Doppler ultrasound compression test is a comparable and important equivalent test for the diagnosis of a venous malformation. With correct color Doppler ultrasound settings, the signal inside the blood- filled caverns shows a change from red to blue and vice versa during transducer pressure/release of pressure on the malformation. No flow is detected in approximately 15-20\% of lesions; this may be due to either thrombosis, substantially low flow inside the malformation, or due to inappropriate Doppler settings. Venous malformations do not harbour arteriovenous shunts, therefore, it is important to search for regions with aliasing 
(turbulence) using color Doppler ultrasound and for shunt flow with spectral wave analysis. If a shunt is detected, the lesion is by definition not a venous malformation but rather an arteriovenous malformation and this is an important distinction in terms of treatment planning. ${ }^{8}$

Simple macrocystic lymphatic malformations are septate caverns of large size filled with anechoic fluid. Bleeding or infection may sometimes result in mixed echogenity of fluid in different caverns and/or the presence of debris layered at the bottom of the caverns. Typically the caverns themselves are not compressible with the transducer. Using color Doppler ultrasound, small arteries or veins may be found inside cyst walls or the surrounding stroma of mere lymphatic malformations. However, cysts themselves are not vascularized at all, unless a combined venolymphatic malformation is present. Only slow flow is detected inside veins and arteries of lymphatic malformations using spectral wave analysis. ${ }^{8,13,14}$

On sonography, lipomas present as well defined oval or round-shaped structures that follow the axis of the skin layers. Fibrolipomas tend to appear as hypoechoic whereas angiolipomas appear hyperechoic and heterogeneous. Hyperechoic fibrous septa can frequently be detected within the tumors. They commonly show hypovascularity on color Doppler ultrasound, and when hypervascularity is detected in a lipoma, an atypical or malignant transformation should be ruled out. ${ }^{15,16,17}$

Hemangiomas differ in their appearance according to the phase; thus, lesions in the proliferative stage appear as hypoechoic hypervascular ill-defined structures on ultrasound. Most hemangiomas show blood flow through many small vessels that may not be detectable with B-mode ultrasound. Therefore, on color Doppler ultrasound, hemangiomas are typically hypervascular with intense perfusion and high vessel density (five or more vessel counts per square centimeter). With duplex ultrasound the arterial vessels inside a hemangioma have a Doppler shift of $>2$ $\mathrm{KHz}$, or in other words show high systolic flow velocities. Proliferative hemangiomas show prominent arterial blood flow, low velocity venous vessels, and occasionally arteriovenous shunts. During the involution phase, the arteriovenous shunts start to disappear and the peak systolic velocity of the arterial vessels tends to decrease. ${ }^{8,18,14,19}$

Localized neurofibromas appear on ultrasound as round, ovoid, or fusiform-shaped hypoechoic nodules. There are variable degrees of vascularity reported in localized neurofibromas on color Doppler ultrasound that can vary from hypo- to hypervascular and neurofibromas can also be associated with hemorrhagic episodes. Plexiform neurofibromas involve branches of nerves that can adopt a serpentine shape that has been also called "bag of worms". Multiple and tortuous hypoechoic tracts following the course of a neural branch can be detected using sonography. These tracts are frequently hypovascular on color Doppler ultrasound. Diffuse neurofibromas present as ill-defined plaque-like lesions with mixed echogenicity (hyperechoic and hypoechoic areas) sometimes with multiple hypoechoic tubule-like and tortuous tracts or nodules. On color Doppler ultrasound, vascularity may vary from hypo- to hypervascular. ${ }^{8}$
USG may help in determining the best treatment approach by preoperative assessment of cutaneous neoplasms, usefully integrating clinical findings, and is also helpful in the follow-up of the patients after surgery, cryotherapy, or laser treatment. ${ }^{4,8,20}$

Francesco Giovagnorio et al evaluated 71 consecutive visible and palpable nodules of the skin from 51 patients. They found the four patterns of vascularity. These observations may suggest that the type IV pattern (hypervascular with internal vessels) is typical of a malignant tumor without necrosis, whereas the type III (hypervascular with multiple peripheral poles but no internal vessels) corresponds to a tumor with central necrosis. The type I (avascular nodule), on the other hand, is the least specific because it can be observed both in totally necrotic malignant tumors and in benign tumors (which are generally vascularized by one or two small peripheral arteries with a perfusion pressure too low to be effectively detected), so concluded that, color Doppler was able to increase significantly the specificity of sonography in the evaluation of skin nodules, but the availability of well-known and affordable gold standards, such as physical examination and biopsy, will probably limit its diagnostic role to some well-defined fields. ${ }^{3}$

Basal cell carcinoma has been characterized as masses of epidermis, dermis and subcutaneous cellular tissue which are hypoechoic with irregular edges and increased vascularity. ${ }^{21}$ On sonography, melanomas usually appear as well defined, oval- or fusiform-shaped, homogeneous, hypoechoic lesions with smooth borders, increased acoustic transmission, and variable degrees of vascularity, even though they commonly show hypervascularity on color Doppler imaging. Sonography has been proved useful for discriminating melanomas thicker and thinner than $1 \mathrm{~mm}$ which is relevant for deciding the performance of a sentinel node procedure. The vessels are commonly located within the lesion and may also be tracked using contrast-enhanced ultrasound. Assessment of the vascularity, including the peak systolic velocity of the arterial vessels, may provide an idea of the angiogenic power of the tumor that can correlate with the metastatic potential. In cases with ulcerations, the epidermis can show irregularities or discontinuities, and increased echogenicity in the subcutaneous tissue may also be found. Because melanomas can show asymmetry in their thickness, the sonographic measurements should be performed at the deepest point. In the vicinity of the primary lesion, satellite lesions (arising within $2 \mathrm{~cm}$ from the primary tumor) or in transit metastasis (arising greater than $2 \mathrm{~cm}$ away from the primary tumor) can be detected. Usually, there is increased vascularity within the metastases on color Doppler imaging, although the presence of internal vessels can show a variable appearance that can go from hypo- to hypervascular. Occasionally, these metastases can present an irregular appearance and anechogenicity and can be sonographically misdiagnosed as abscesses. This challenging anechoic appearance has been reported as related more to hypercellularity and not as a result of necrosis. The balloon shape, nodular thickening of the cortex, and loss of hyperechogenicity of the medullae are signs of malignant infiltration in regard to nodal infiltration. The use of lower frequency probes in bulky primary melanomas may help 
to reach to the bottom of the lesion and provide a better definition of the extension. $8,22,23,24,25,26$

\section{CONCLUSION}

HRUS is non-invasive, affordable, easily available, rapid imaging technology. It can be used to localize the skin lesion, generate differential diagnosis and to follow up known lesion. Differentiation of skin lesions is possible on the basis of echogenicity, margins, vascularity of the lesion but for confirmation of the diagnosis clinical features, histopathological examination is needed. Though HRUS helped in differentiating the benign from malignant lesion, particular HRUS findings did not help in the diagnosis of lesion. So, large study with more sample size is needed to find the particular HRUS findings.

\section{REFERENCES}

1. Mandava A, Ravuri PR, Konathan R. High-resolution ultrasound imaging of cutaneous lesions. Indian J Radiol Imaging 2013;23(2): 269-77

2. Elisa de Oliveira Barcaui, Antonio Carlos Pires Carvalho, Flavia Paiva Proença Lobo Lopes, Juan PiñeiroMaceira, Carlos Baptista Barcaui. High frequency ultrasound with color Doppler in dermatology. An Bras Dermatol. 2016; 91(3): 262-273.

3. Francesco Giovagnorio, Chiara Andreoli, Maria Luisa De Cicco. Color Doppler Sonography of Focal Lesions of the Skin and Subcutaneous Tissue. J Ultrasound Med. 1999;18(2):89-93.

4. Radu B, Maria C, Monica L, Lucian F. Diagnosis and characterization of cutaneous tumors using combined ultrasonographic procedures (conventional and high resolution ultrasonography) Med Ultrason. 2010;12 (4):317-22.

5. Harland C, Bamber JC, Gusterson B, Mortimer P. High-frequency,high resolution B-scan ultrasound in the assessment of skin tumours. Br J Dermatol 1993;128 (1):525-32.

6. Machet L, Samimi M, Georgesco G, Mourtada Y, Naouri M, Grégoire JM, et al.High resolution ultrasound imaging of melanocytic and other pigmented lesions of the skin. Ultrasound Imaging, Masayuki Tanabe (Ed.), ISBN: 978-953-307-239-5, InTech.

7. G Ravichandra, Shankar Arjun, Shad Ajmal, Shenoy Manjunath, Shamseena Ayshath. High resolution ultrasonography in dermatology; a psoriasis experience. Indian Journal of Basic and Applied Medical Research; 2016:5(2):121-125.

8. Ximena Wortsman, Gregor B.E. Jemec. Dermatologic Ultrasound with Clinical and Histologic Correlations, Springer publication, 2013.

9. Tayal VS, Hasan N, Norton HJ, Tomaszewski CA.The effect of soft-tissue ultrasound on the management of cellulitis in the emergency department. Acad Emerg Med. 2006;13(4):384-8.

10. Papadimitriou CS, Kittas CN. Normal structure and function of lymph nodes. In: Pangalis GA and Polliack A, eds. Benign and malignant lymphadenopathies, Chur: Harwood Academic Publishers, 1993:113-30.

11. Ying TC,Ultrasound evaluation of cervical lymph nodes in a Chinese population, in M.Phil. Thesis, Department of Optometry and Radiography. 1996, The Hong Kong Polytechnic University: Hong Kong. p. 235.

12. Evans RM, Ahuja A, Metreweli C. The linear echogenic hilus in cervical lymphadenopathy-a sign of benignity or malignancy? Clin Radiol 1993;47 (5):262-4.

13. Wong KT, Lee YY, King AD, Ahuja AT. Imaging of cystic or cyst-like neck masses. Clin Radiol 2008; 63 (6): 613-22.

14. Abernathy L. Paediatric neck lumps III - Vascular and lymphatic malformations. Ultrasound. 2007;15(1): 1427.

15. Kumar V, Abbas AK, Fausto N et-al. Robbins and Cotran pathologic basis of disease. W B Saunders Co. (2005) ISBN:0721601871.

16. DiDomenico P, Middleton W. Sonographic evaluation of palpable superficial masses. Radiol. Clin. North Am. 2014;52(6): 1295305.

17. Inampudi P, Jacobson JA, Fessell DP, Carlos RC, Patel SV, Delaney-Sathy LO, van Holsbeeck MT. Soft-tissue lipomas: accuracy of sonography in diagnosis with pathologic correlation. Radiology. 2004;233 (3): 763-7.

18. Donnelly LF, Adams DM, Bisset GS. Vascular malformations and hemangiomas: a practical approach in a multidisciplinary clinic. AJR Am J Roentgenol 2000; 174 (5): 597-608.

19. Dubois J, Alison M. Vascular anomalies: What a radiologist needs to know. Pediatr Radiol 2010;40 (1):895-905.

20. Cammarota T, Pinto F, Magliaro A, Sarno A. Current uses of diagnostic high-frequency US in dermatology. Eur J Radiol 1998;27 (4):215-23.

21. Cammarota T, Pinto F, Magliaro A, Sarno A. Current uses of diagnostic high-frequency US in dermatology. Eur J Radiol 1998;27 (6):215-23.

22. Desai TD, Desai AD, Horowitz DC, Kartono F, WahlT. The use of high frequency ultrasound in the evaluation of superficial and nodular basal cell carcinomas. Dermatol Surg 2007; 33 (2): 1226-1227.

23. Jovanović D, Paravina M, Spalević L, Stanojević M, Tiodorović J, Binić I, et al. Characteristics of malignant melanoma examined by $20-\mathrm{MHz}$ ultrasound. Facta Univ Med Biol 1998;5 (1):58-60.

24. Alexander H, Miller DL. Determining skin thickness with pulsed ultrasound. J Invest Dermatol 1979;72 (5):179.

25. Nazarian LN, Alexander AA, Rawool NM, Kurtz AB, Maguire HC, Mastrangelo MJ. Malignant melanoma: impact of superficial US on management. Radiology. 1996;199 (3):273-7.

26. Ekwueme DU, Guy G, Li C, Rim SH, Parelkar P, Chen SC. The health burden and economic costs of cutaneous melanoma mortality by race/ethnicityUnited States, 2000 to 2006. J Am Acad Dermatol. 2011;6(S1):S133-43.

27. Ruocco E, Argenziano G, Pellacani G, Seidenari S. Noninvasive imaging of skin tumors. Dermatol Surg 2004; 30 (1): 301-310.

\section{Source of Support: Nil; Conflict of Interest: None}

Submitted: 12-10-2018; Accepted: 13-11-2018; Published online: 25-11-2018 